DOI: 10.12731/wsd-2018-4-134-144

УДК 615.85

\title{
РЕЗУЛЬТАТЫ СТАБИЛОМЕТРИИ ПРИ ПРИМЕНЕНИИ ВОЙТА-ТЕРАПИИ У ДЕТЕЙ С ДЦП
}

\author{
Тучков В.Е., Киселев Д.А.
}

Целью исследования явилось научное обоснование эффективности применения восстановительных мероприятий с применением метода Войта-терапии для коррекции двигательных нарушений при ДЦП.

Материалы и методы. В исследовании принимали участие 64 ребенка обоего пола в возрастном интервале от 1 до 3 лет с диагнозом: ДЦП, гемипаретическая форма поражения, которые проходили восстановительное лечение на кафедре реабилитации и спортивной медицины РНИМУ им. Н.И. Пирогова. Пациенты были распределены на две группы по 32 человека. В экспериментальной группе для восстановительного лечения детей комплексно применялись методы кинезиотейпирования и Войта-терапии.

Результаты исследования. В представленной работе рассматриваются результаты исследования эффективности применения комплексной методики реабилитации на основе улучшения показателей координаторных способностей детей с гемипаретической формой ДЦП

Выводы. Полученные данные в цеелом демонстрируют положительную динамику применения комплексной методики реабилитации у детей с гепипаретической формой ДЦП на основе сочетания методов Войта-терапии и кинезиотейпирования.

Ключевые слова: методика; Войта-терапия; аутохтонная мускулатура; ДЦП.

\section{THE RESULTS OF THE APPLICATION OF COMPLEX METHODS OF REHABILITATION IN CHILDREN WITH CEREBRAL PALSY}

\author{
Tuchkov V. E., Kiselev D.A.
}

The aim of the study was scientific substantiation of the effectiveness of remediation using the method of Vojta therapy for the correction of motor disorders in cerebral palsy. 
Materials and methods. The study involved 64 children of both sexes in the age interval from 1 to 3 years diagnosis: cerebral palsy, hemiparetic form of defeat, which took place rehabilitation treatment at the Department of rehabilitation and sports medicine, Russian national research medical University them. N. And. Pirogov. The patients were divided into two groups of 32 people. In the experimental group for restorative treatment of children comprehensively we have applied the methods of kinesiotherapy and Vojta therapy.

The results of the study. In the presented work considers results of research of efficiency of application of complex methods of rehabilitation based on improved indicators coordinatory skills of children with hemiparetic form of cerebral palsy.

Conclusions. Overall, the data received demonstrate positive dynamics application for integrated methods of rehabilitation in children with hemiparetic form of cerebral palsy on the basis of a combination of the methods of Vojta therapy and kinesiotherapy.

Keywords: technique; Vojta therapy; autochthonous muscles; cerebrel palsy.

\section{Актуальность}

Гемипаретическая форма ДЦП остается одной из распространенных и трудных для восстановительного лечения заболеваний $[3$, с. 103 , 4, с. 72]. У значительного числа больных (около 30\%) уже на ранних этапах формируются порочные позы, контрактуры и патологические двигательные стереотипы. Специалисты предлагают различные методики восстановительного лечения детей с ДЦП (гемипаретическая форма), однако результаты не обеспечивают высокого реабилитационного эффекта. Частая диагностика синдрома, тяжесть клинических проявлений и появление новых методик подчеркивают актуальность проблемы восстановления детей, страдающих гемипаретической формой ДЦП.

Одним из современных методов восстановления таких больных в настоящее время является Войта-терапия [3, с. 75, 5, с. 20, 6, с. 174]. Однако в доступной нам литературе мы не нашли разработанных методов восстановительного лечения детей от 1 года до 3 лет с гемипаретической формой ДЦП с использованием данного метода.

Целью исследования явилось научное обоснование эффективности применения восстановительных мероприятий с применением метода Войта-терапии для коррекции двигательных нарушений при ДЦП. 


\section{Описание методики}

Основной методикой в комплексной реабилитации детей с ДЦП мы использовали Войта-терапию. Войта терапия - способ лечения детей с различными формами поражения нервной системы, разработанный в середине XX века чешским неврологом Вацлавом Войтой $[3$, с. 75,5, с. 20 , 6, с. 174]. Данный способ реабилитации активно использовался нами на базе кафедры реабилитации и спортивной медицины РНИМУ им. Н.И. Пирогова.

Применение методики кинезиотейпирования базировалось на практических и теоретических подходах, разработанных Д.А. Киселевым [9, с. 77,10 , с. 28,12 , с. 110], основанных на теории метода РДА [13, с. 49], применяемых на базе кафедры реабилитации и спортивной медицины РНИМУ им. Н.И. Пирогова.

Работая с детьми, имеющими различные поражения нервной системы, мы пришли к выводу, что минимальная стимуляция рецепторного аппарата кожных покровов, подкожно-жировой клетчатки, поверхностной фасции передней поверхности шеи приводит к включению функций центрирования головы, шейного отдела и всего тела, основанной на активации аутохтонной мускулатуры на фоне изменения рецепции.

Для кинезиотейпирования, направленного на включение функции центрирования были использованы различные по своим эластическим свойствам тейпы $[8$, с. 138,9 , с. 67,11, с. 15], позволяющие целенаправленно активировать проприоцептивный аппарат пациента. Это приводило к формированию новой функциональной системы регуляции движения и коррекции паталогического состояния пациента, а в условиях применения дополнительных методов, значительно увеличивало глубину и эффективность их воздействия.

\section{Организация исследования}

В исследовании принимали участие 64 ребенка обоего пола в возрастном интервале от 1 до 3 лет с диагнозом: ДЦП, гемипаретическая форма поражения, которые проходили восстановительное лечение на кафедре реабилитации и спортивной медицины РНИМУ им. Н.И. Пирогова. Пациенты были распределены на две группы по 32 человека. В экспериментальной группе для восстановительного лечения детей комплексно применялись методы кинезиотейпирования и Войта-терапии. 
В контрольной группе реабилитация проводилась с использованием метода Войта-терапии.

\section{Методы исследования}

Говоря о методах исследования, мы акцентируем внимание на том, что большинство рассматриваемых двигательных навыков (нарушений) таковы, что полностью отсутствует потребность в их специальной оценке и введении в методы исследования каких-либо инструментальных оценок и (или) клинических тестов с целью демонстрации положительной динамики.

У пациентов, с которыми это было возможно - стабилометрический контроль параметров постурологической регуляции $[8$, с. 43,11, с. 23,13 , c. 630,14, c. 50$]$.

В этой связи основными методами исследования были анализ специальной литературы и динамические наблюдения (клинический осмотр) пациентов с визуальной оценкой степени патологических нарушений в период восстановительного лечения.

\section{Методика работы}

Упражнения по методике Войта-терапии проводились в следующей последовательности:

1) Упражнение на основе рефлекторного поворота со спины на бок

2) Упражнение на основе рефлекторного поворота с бока на живот

3) Упражнение на основе рефлекторного ползания

\section{Результаты исследования}

Показателем положительной динамики применения данной методики являлось приобретение многочисленных навыков моторной активности, ранее отсутствовавших. К ним относятся в первую очередь:

- исчезновение асимметрии положения туловища;

- появление опороспособности на руки (на локтевые суставы, на сами кисти);

- появление способности стойки на четвереньках, ползания на животе, ходьбы на четвереньках, появление способности к вертикализации, первичной ходьбе;

- уменьшение (исчезновение) асимметрии между здоровыми и пораженными конечностями; и мн. др.

Графические результаты стабилометрического исследования 
Таблича 1.

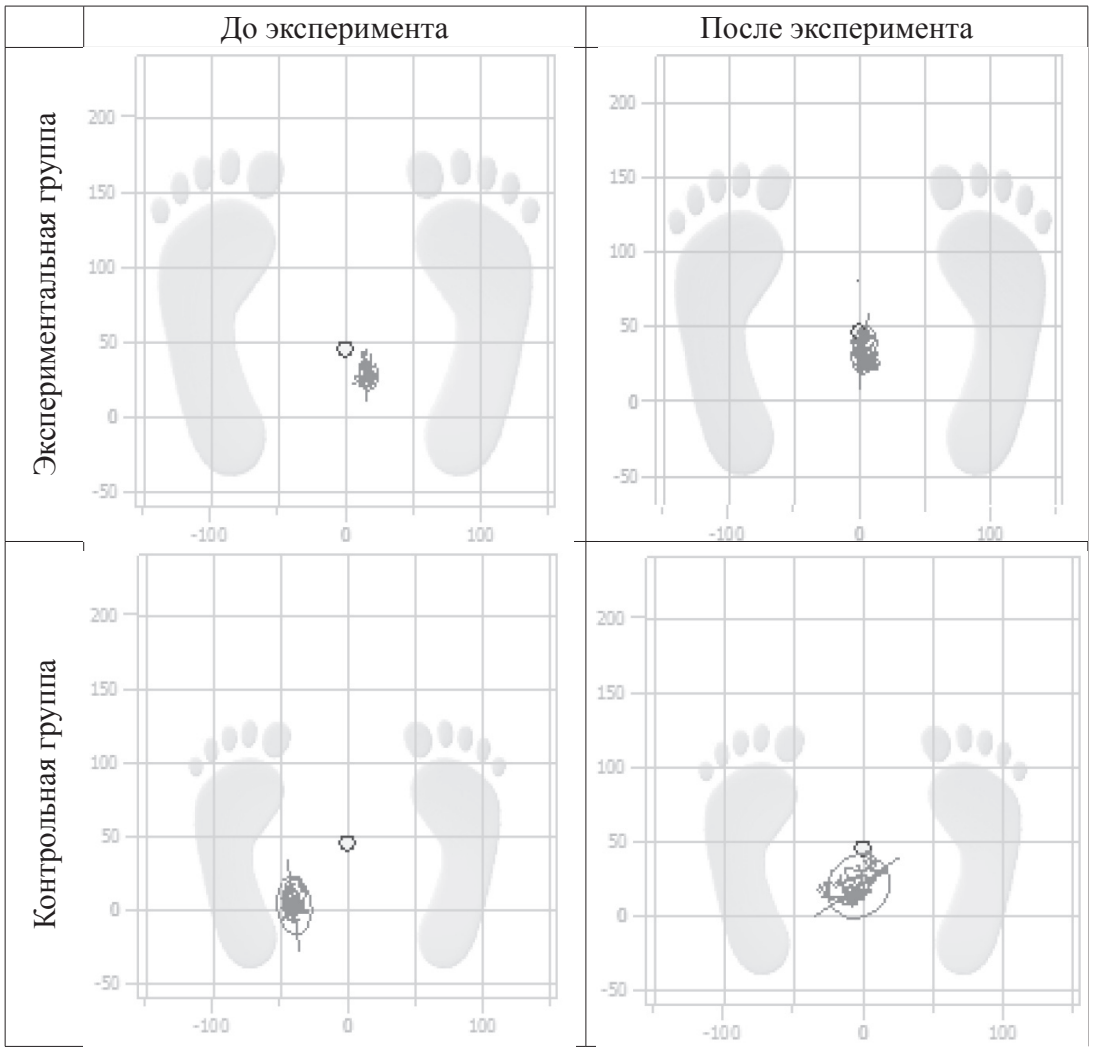

Сравнительные данные стабилометрических показателей контрольной и экспериментальной группы до и после эксперимента. $(\mathrm{M} \pm \sigma)$

Таблицуа 2.

\begin{tabular}{|l|c|c|c|c|}
\hline & \multicolumn{2}{|c|}{$\begin{array}{c}\text { Экспериментальная } \\
\text { группа }\end{array}$} & \multicolumn{2}{c|}{$\begin{array}{c}\text { Контрольная } \\
\text { группа }\end{array}$} \\
\hline $\begin{array}{l}\text { Среднее положение } \\
\text { ОЦД во фронтальной } \\
\text { плоскости Х (мм) }\end{array}$ & $\begin{array}{c}\text { До экспери- } \\
\text { мента }\end{array}$ & $\begin{array}{l}\text { После экс- } \\
\text { перимента }\end{array}$ & $\begin{array}{l}\text { До экспери- } \\
\text { мента }\end{array}$ & $\begin{array}{c}\text { После экс- } \\
\text { перимента }\end{array}$ \\
\cline { 2 - 5 } & $16,9 \pm 1,5$ & $4,89 \pm 3,8 *$ & $-40 \pm 1,3$ & $3,67 \pm 2,1$ * \\
\hline $\begin{array}{l}\text { Среднее положение } \\
\text { ОЦД в сагиттальной } \\
\text { плоскости Ү(мм) }\end{array}$ & $27,8 \pm 1,9$ & $15,6 \pm 1,3 *$ & $3,75 \pm 1,2$ & $20,5 \pm 1,3 *$ \\
\hline
\end{tabular}


Окончание табл. 1.

\begin{tabular}{|l|c|c|c|c|}
\hline $\begin{array}{l}\text { Среднеквадратическое } \\
\text { отклонение ОЦД во } \\
\text { фронтальной плоско- } \\
\text { сти Мах Х (мм) }\end{array}$ & $10,2 \pm 1,7$ & $8,91 \pm 1,1$ & $10,7 \pm 1,2$ & $5,7 \pm 3,18$ \\
\hline $\begin{array}{l}\text { Среднеквадратическое } \\
\text { отклонение ОЦД в са- } \\
\text { гиттальной плоскости } \\
\text { Мах Ү (мм) }\end{array}$ & $42 \pm 1,8$ & $46,5 \pm 1,3$ & $11,4 \pm 3,2$ & $19,6 \pm 1,7$ \\
\hline $\begin{array}{l}\text { Скорость ОЦД V } \\
\text { (мм/с) }\end{array}$ & $9,66 \pm 1,3$ & $9,54 \pm 0,8$ & $9,51 \pm 0,8$ & $6,18 \pm 0,5$ \\
\hline $\begin{array}{l}\text { Площадь статокинези- } \\
\text { ограммы S (мм })\end{array}$ & $94,4 \pm 1,9$ & $86,8 \pm 1,3$ & $161 \pm 1,4$ & $98,1 \pm 3.8$ \\
\hline $\begin{array}{l}\text { Индекс стабильности } \\
\text { Si (\%) }\end{array}$ & $41,4 \pm 1,6$ & $43,5 \pm 1,1$ & $42 \pm 1,7$ & $48,9 \pm 0,7$ \\
\hline Энергоиндекс Еі (Дж) & $5,3 \pm 1,7$ & $4,8 \pm 2,4$ & $3,59 \pm 1,5$ & $1,76 \pm 2,3$ \\
\hline
\end{tabular}

Как видно из исследований отмечается выраженная центрация пациента $(\sim \mathrm{X}: 16,9 \rightarrow 4,89)$, увеличение опороспособности на пяточную область (уменьшение эвинусной установки стопы): $(\sim$ Y: $27,8 \rightarrow 15,6)$; выраженная стабилизация и увеличение устойчивости: уменьшение «Среднеквадратического отклонения ОЦД во фронтальной плоскости» и включение глубокой регуляции основной стойки: увеличение «Среднеквадратического отклонения ОЦД в сагиттальной плоскости». Исчезновение сгибательной установки левого коленного сустава, выраженное уменьшение сгибательной установки левого тазобедренного сустава, выраженное уменьшение эквинусной установки левой стопы при ходьбе.

При заданной вероятности $\mathrm{p}<0,05$ с применением критерия Стъюдента мы доказали достоверность различий в экспериментальной и контрольной группах следующих координаторных проб:

1) Среднее положение ОЦД в фронтальной плоскости;

2) Среднее положение ОЦД в саггитальной плоскости.

Все остальные пробы также имели положительную динамику, ярче выраженную в экспериментальной группе, но различия между ними по критерию Стъюдента достоверными не оказались.

\section{Обсуждение}

Сочетание методик кинезиотейпирования с Войта-терапией привело к расширению практического применения Войта-терапии с целью более интенсивного воздействия на аутохтонную мускулатуру шейного, грудного и поясничного отделов. Немаловажно то, что такое сочетание методик 
стало применяться нами на детях данного возраста с различными проблемами и заболеваниями ранее, чем у детей с гемипаретической формой ДЦП. И именно они стали первыми пациентами, которые показали, что такое применение Войта-терапии приводит к выраженным результатам включения функции центрирования, улучшения осанки и даже улучшения походки. В дальнейшем все это было подтверждено положительной динамикой у детей первых лет жизни.

По многочисленным свидетельствам врачей-специалистов, родителей, которые выполняли данную методику после наложения тейпов, в течение курса совмещения тейпирования и Vojta-терапии наблюдаются следующие изменения:

1) Выраженное улучшение рефлекторного ответа при выполнении методики.

2) Расширение двигательных возможностей ребенка с последующей потенциацией при дальнейшей работе.

3) Изменение (усложнение) выполнения метода, вследствие увеличения двигательных возможностей пациента.

4) Присоединение к методике Vojta-терапии других реабилитационных подходов, выполнение которых ранее было физически невозможно.

\section{Выводы}

1) Использование комплексной методики реабилитации способствует улучшению показателей среднего положение ОЦД в фронтальной плоскости и среднее положение ОЦД в саггитальной плоскости.

2) Сочетание Войта-терапии с кинезиотейпированием значительно улучшает работу на стабилоплатформе при ДЦП (гемипаретическая форма).

3) Преимуществом сочетания данных методик является быстрое формирование правильного двигательного стереотипа, что способствует увеличению эффективности мероприятий восстановительного лечения и снижению количества оперативных вмешательств.

4) Применение комплексной методики реабилитации, направленной на включение функции центрирования приводит к симметричной работе аутохтонной мускулатуры позвоночника, устраняя при этом асимметричный мышечный тонус.

\section{Сиисок литературы}

1. Авакян Р.К. Применение биотренинга по стабилограмме в комплексном лечении больных паркинсонизмом: автореф. ...дис. канд. мед. наук : 03.00.13. М., 2001. 213 c. 
2. Бадалян Л.О. Детская неврология: учебное пособие. М.: МЕДпрессинформ, 2001. $608 \mathrm{c}$.

3. Бадалян Л.О. Детские церебральные параличи / Л.О. Бадалян, Л.Г. Журба, О.В. Тимонина. Киев, 1988.

4. Бадалян Л.О. К проблеме детских церебральных параличей / Л.О. Бадалян, Г.Н. Дунаевская, И.А. Скворцов // Вести АМН СССР. 1983. № 6. С. 71-79.

5. Войта-терапия в раннем детском возрасте: методические рекомендации международного общества Войто-терапевтов. Прага, 1992.

6. В. Войта. Принцип Войта / В. Войта, А. Петерс. Springer, 2007. 171 с.

7. Киселев Д.А. Анализ результатов инструментальных методов исследования клинического применения метода физиологической регуляции движения / Д.А. Киселев, В.А. Балабанова, О.А. Лайшева // Лечебная физкультура и спортивная медицина. 2011. №7 (91). С. 41-51.

8. Киселев Д.А. Применение кинезиотейпирования при нарушениях функций центрирования, ассиметричном мышечном тонусе у детей первого года жизни - MEDICUS / Д.А. Киселев, В.Е. Тучков, В.В. Губанов // Международный медицинский научный журнал. 2016. №1 (7). С. 136-141.

9. Киселев Д.А. Реабилитация детей с ДЦП с использованием метода кинезиотейпирования MEDICUS / Д.А. Киселев, В.Е. Тучков, В.В. Губанов // Международный медицинский научный журнал. 2015. №5 (5). С. 65-70.

10. Кармазин В.В., Киселев Д.А., Кузин В.В., Лайшева О.А., Поляев Б.А. Реабилитация детей с неврологическими заболеваниями с использованием стабилометрии и метода ликвидации патологической синергии // Научно-практический медицинский журнал «Доктор,Ру». №5, 2007. С. 22-29.

11. Киселев Д.А. Кинезиотейпинг в лечебной практике неврологии и ортопедии. СПб: Питер, 2015. 168 с.

12. Киселев Д.А., Лайшева О.А., Губанов В.В. Реабилитация больных с поражением спинного мозга с использованием метода функциональной регуляции движения // Саратовский научно-медицинский журнал. 2010. Том 6, №3 июль-сентябрь. С. 628-632.

13. Киселев Д.А., Лайшева О.А., Фрадкина М.М. Реабилитация больных с поражением ЦНС с использованием метода функциональной регуляции движения // Научно-практический журнал «Детская Больница», №4 (42), 2010. С. 48-55.

14. Реабилитация детей с неврологическими заболеваниями с использованием стабилометрии и метода ликвидации патологической синергии / В. В. Карамзин [и др.] // Доктор.Ру. М., 2007. №5. С. 22-29.

15. Семенова К.А. Восстановительное лечение больных с резидуальной стадией детского церебрального паралича. М.: Антидор, 1999. 384 с. 
16. Тучков В.Е. Влияние кинезиотейпирования на функциональное состояние коленного сустава высококвалифицированных баскетболистов // Университетский спорт: Здоровье и процветание нации: материалы $\mathrm{V}$ международной научной конференции студентов и молодых ученых: в 2 т. Казань, 2015. T. 1. C. $268-333$.

17. The effects of Kinesio Taping on body functions and activity in unilateral spastic cerebral palsy: A single-blind randomized controlled trail / Ozgun Kaya Kara [etc.]; Department of physiotherapy and rehabilitation ; Department of occupational therapy // Developmental medicine and child neurology. Ankara, 2014. P. 1.

18. The effect of Kinesio Taping on proprioception at the ankle / Travis Halseth [etc.] // Journal of Sports Science and Medicine. 2004. № 3, pp. 1-7.

19. The effects of Taping Prior to PNF treatment on lower extremity proprioception of hemiplegic patients / Yong-Kyu Choi [etc.] // Journal of Physical Therapy Science. 2013. № 25, pp. 1119-1122.

20. Vojta V. Die cerebralen Bewegungsstorungen im Sagligsalter. Stuttgart: Tieme, Verlag, 1981. $243 \mathrm{p}$.

\section{References}

1. Avakyan R.K. Primenenie biotreninga po stabilogramme v kompleksnom lechenii bol'nykh parkinsonizmom [Application of biotraining on stabilogram in complex treatment of patients with parkinsonism]. M., 2001. 213 p.

2. Badalyan L.O. Detskaya nevrologiya [Pediatric Neurology]: A Training Manual. Moscow: MEDPressinform, 2001. $608 \mathrm{p}$.

3. Badalyan L.O., Zhurba L.G., Timonina O.V. Detskie tserebral'nye paralichi [Children's cerebral palsy]. Kiev, 1988.

4. Badalyan L.O., Dunaevskaya G.N., Skvortsov I.A. K probleme detskikh tserebral'nykh paralichey [To the problem of children's cerebral paralysis]. Vesti AMN SSSR [News of the Academy of Medical Sciences of the USSR]. 1983. No. 6, pp. 71-79.

5. Voyta-terapiya $v$ rannem detskom vozraste: metodicheskie rekomendatsii mezhdunarodnogo obshchestva Voyto-terapevtov [Vojta-therapy in early childhood: methodical recommendations of the international society Vojto-therapists]. Prague, 1992.

6. Vojta V., Peters A. Printsip Voyta [The principle of Vojta]. Springer, 2007. 171 p.

7. Kiselev D.A., Balabanova V.A., Laysheva O.A. Analiz rezul'tatov instrumental'nykh metodov issledovaniya klinicheskogo primeneniya metoda fiziologicheskoy regulyatsii dvizheniya [Analysis of the results of instrumental methods for investigating the clinical application of the method of physio- 
logical regulation of motion]. Lechebnaya fizkul'tura i sportivnaya meditsina [Therapeutic physical training and sports medicine]. 2011. № 7 (91). P. 41-51.

8. Kiselev D.A., Tuchkov V.E., Gubanov V.V. Primenenie kinezioteypirovaniya pri narusheniyakh funktsiy tsentrirovaniya, assimetrichnom myshechnom tonuse $\mathrm{u}$ detey pervogo goda zhizni - MEDICUS [Use kinesiotyepirovaniya in violation of the functions of centering, asymmetric muscle tone in children of the first year of life - MEDICUS]. Mezhdunarodnyy meditsinskiy nauchnyy zhurnal [International Medical Scientific Journal]. 2016. № 1 (7), pp. 136-141.

9. Kiselev D.A., Tuchkov V.E., Gubanov V.V. Reabilitatsiya detey s DTsP s ispol'zovaniem metoda kinezioteypirovaniya MEDICUS [Rehabilitation of children with cerebral palsy using the kinesiotherapy method MEDICUS]. Mezhdunarodnyy meditsinskiy nauchnyy zhurnal [International Medical Scientific Journal]. 2015. № 5 (5), pp. 65-70.

10. Karmazin V.V., Kiselev D.A., Kuzin V.V., Laysheva O.A., Polyaev B.A. Reabilitatsiya detey s nevrologicheskimi zabolevaniyami s ispol'zovaniem stabilometrii i metoda likvidatsii patologicheskoy sinergii [Rehabilitation of children with neurological diseases using stabilometry and the method of elimination of pathological synergy]. Doctor.Ru. № 5, 2007, pp. 22-29.

11. Kiselev D.A. Kinezioteyping v lechebnoy praktike nevrologii i ortopedii [Kinesiotherapy in the medical practice of neurology and orthopedics]. St. Petersburg: Peter, 2015. $168 \mathrm{p}$.

12. Kiselev D.A., Laysheva O.A., Gubanov V.V. Reabilitatsiya bol'nykh s porazheniem spinnogo mozga s ispol'zovaniem metoda funktsional'noy regulyatsii dvizheniya [Rehabilitation of patients with spinal cord injury using the method of functional regulation of motion]. Saratovskiy nauchno-meditsinskiy zhurnal [Saratov Journal of Medical Scientific Research]. 2010. Volume 6, No. 3 July-September, pp. 628-632.

13. Kiselev D.A., Laysheva O.A., Fradkina M.M. Reabilitatsiya bol'nykh s porazheniem TsNS s ispol'zovaniem metoda funktsional'noy regulyatsii dvizheniya [Rehabilitation of patients with CNS damage using the method of functional regulation of motion]. Detskaya Bol'nitsa [Children’s Hospital], №4 (42), 2010, pp. 48-55.

14. Karamzin V.V. et al. Reabilitatsiya detey s nevrologicheskimi zabolevaniyami s ispol'zovaniem stabilometrii i metoda likvidatsii patologicheskoy sinergii [Rehabilitation of children with neurological diseases using stabilometry and the method of elimination of pathological synergy]. Doktor:Ru. M., 2007. №5, pp. 22-29.

15. Semenova K. A. Vosstanovitel'noe lechenie bol'nykh s rezidual'noy stadiey detskogo tserebral'nogo paralicha [Rehabilitation treatment of patients with residual stage of cerebral palsy]. Moscow: Antidor, 1999. 384 p. 
16. Tuchkov V. E. Vliyanie kinezioteypirovaniya na funktsional'noe sostoyanie kolennogo sustava vysokokvalifitsirovannykh basketbolistov [Influence of kinesiotherapy on the functional state of the knee of highly qualified basketball players]. Universitetskiy sport: Zdorov'e i protsvetanie natsii : materialy V mezhdunarodnoy nauchnoy konferentsii studentov i molodykh uchenykh [University sport: Health and prosperity of the nation: materials of the V International Scientific Conference of Students and Young Scientists]. Kazan, 2015. V. 1, pp. 268-333.

17. The effects of Kinesio Taping on the body functions and activity in unilateral spastic cerebral palsy: A single-blind randomized controlled trail / Ozgun Kaya Kara [etc.]; Department of physiotherapy and rehabilitation; Department of occupational therapy. Developmental medicine and child neurology. Ankara, 2014. P. 1.

18. Travis Halseth [etc.] The effect of Kinesio Taping on proprioception at the ankle. Journal of Sports Science and Medicine. 2004. № 3, pp. 1-7.

19. Yong-Kyu Choi [etc.] The Effects of Taping Prior to PNF treatment. Journal of Physical Therapy Science. 2013. No. 25, pp. 1119-1122.

21. Vojta V. Die cerebralen Bewegungsstorungen im Sagligsalter. Stuttgart: Tieme, Verlag, 1981. 243 p.

\section{ДАННЫЕ ОБ АВТОРАХ}

Тучков Владимир Евгеньевич, заведующий лабораторией кафедры физиологии и биохимии, специалист по адаптивной физической культуре ФГБУ ФНЦ ВНИИФ

Елизаветинский пер., 10, стр.1, г. Москва, 105005, РФ tych33@yandex.ru

Киселев Дмитрий Анатольевич, старший научный сотрудник кафедры реабилитации и спортивной медицины, кандидат медицинских наук Российский государственный медицинский университет ул. Островитянова, 1, г. Москва, 117997, РФ

DATA ABOUT THE AUTHORS

Tuchkov Vladimir Evgenievich, Head of the Laboratory of the Department of Physiology and Biochemistry, Specialist in Adaptive Physical Education Federal Science Center for Physical Culture and Sport 10/1, Elizavetinsky per., Moscow, 105005, Russian Federation tych33@yandex.ru

Kiselev Dmitry Anatolyevich, Senior Fellow at the Department of Rehabilitation and Sports Medicine, PhD

Russian State Medical University

1, Ostrovityanov Str., Moscow, 117997, Russian Federation 